\title{
Potential PD-1 / PD-L1 interface acting inhibitors
}

\author{
Thomas W. Comollo, $\mathrm{PhD}^{1}$ \\ ${ }^{1}$ FSDP LLC (Fat Silico Data Pharm) \\ http://fatsilicodatapharm.com
}

Running title: PD-1 interface inhibitors

To whom correspondence should be addressed: Thomas W. Comollo, E-mail: tcomollo@,fatsilicodatapharm.com

Key Words: cancer, Immunotherapy, Programmed Cell Death (PD-1), Programmed Death Ligand 1 (PD-L1), small molecule inhibitors, protein-protein inaction inhibitors, virtual screening

\begin{abstract}
Programmed Cell Death 1 (PD-1) is an immune check point regulating receptor and is a well-known target of immunotherapeutics. PD-1 may be activated through the binding of its ligand, Programmed Death Ligand 1 (PDL1). It has been shown that protein-protein interaction disruption is possible in immune check point modulating receptors, such as TAMs and of the PD-1 / PD-L1 interface. A virtual screening of $\sim 165,000$ compounds was carried out, targeting a crevice in the PD-1 / PD-L1 interface. Small molecule docking using AutoDock Vina was performed against this site because it is proposed that it could cause inhibition of the PD-L1 induced PD-1 activation via interrupting the PD-1 / PD-L1 protein-protein interaction. "Hit" molecules that score well when docked to this pocket may inhibit the PD-1 / PD-L1 interaction.
\end{abstract}

\section{Abbreviations}

Programmed Cell Death 1 (PD-1), Programmed Death Ligand 1 (PD-L1), pocket of interest (POI)

\section{Introduction}

PD-1 is an immune check point regulating receptor expressed on the surface of certain cells. It is a well known target of immunotherapeutics [1,2]. PD-1 activation is involved in down regulating the human body's immune response. The overexpression of PD-L1 on cancer cells and excessive activation PD-1 can also prevent immune response to cancer cells $[3,4]$. Extracellular protein-protein inaction disruption is possible in immune check point modulating receptors, such as TAMs [5] and of the PD-1 / PD-L1 interface [6]. Inhibiting the activation of these receptors in certain cancers may open the cancer cells up to attack by the patient's immune system [4].

A virtual screening of $\sim 165,000$ compounds was carried out, targeting a crevice in the PD-1 / PD-L1 interface. Small molecule docking using AutoDock Vina [7] was performed against this pocket of interest (POI) because it is proposed that it could cause inhibition of the PD-L1 induced PD-1 activation via interrupting the PD-1 / PD-L1 protein-protein interaction. We sought to find "hit" molecules that scored well against our pocket of interest and to make this data available.

\section{Methods}

More information on the protocols used are available at: https://home.fatsilicodatapharm.com/home/virtualscreening-methodology.

\section{Macromolecule model preparation}

The macromolecule model of PD-1 was created using Bioinformatics Toolkit [8], as part of a personal interest 
project while the author was in an academic role. The template use was a crystal structure of PD-1, Protein Data Bank entry, 5wt9.pdb, chain G [9]. The homology model on Bioinformatics Toolkit of the crystal structure of the same protein was created to ensure that all side chains were filled in and that the model was minimized. The model was prepared for docking in AutoDock MGL Tools 1.5.6 [10]. The PD-1 docking model's hydrogen were added, and then merged into nonpolar hydrogen. Gasteiger charges were calculated and AutoDock4 atom types were added. The PD-1 docking model was then saved as a ".pdbqt" file.

\section{Virtual screening ligand preparation}

Clean Drug Like sets 94 and 98 were downloaded from the ZINC12 Database (zinc12.docking.org) [11]. The compressed ".mol2" files were decompressed and then split using Raccoon [12], available from the Olson laboratory, that also produces AutoDock. A Linux script that employs nested "for each" loops and files in AutoDock MGL Tools 1.5.6, based on scripts available in the tutorial available at http://autodock.scripps.edu/faqshelp/tutorial/using-autodock4-for-virtual-screening/UsingAutoDock4forVirtualScreening_v4.pdf, was used to set up ligand files in the proper .pdbqt file for screening. This script also split the ZINC ligand files into 100 folders, numbered 0 to 99, based on the first two numbers in the ZINC Database entry number.

\section{Running the virtual screening}

AutoDock Vina[7] was used for the virtual screening of approximately 165,000 compound ligand files. Each set of the virtual screening, Clean Drug Like 94 and Clean Drug Like 98, were run by a separate instance of a Linux shell script that employed nested "for each" loops that traverse through the folders set up in the "Virtual screening ligand preparation" section to run AutoDock Vina on each, individual, processed ZINC “.pdbqt" ligand file. An 18 by 18 by 18 angstrom search area, centered at 51.831, -63.996, -21.844 on the PD-1 docking model was used as our POI for the screening. Using scripts, log files from each docking were combined, and the best score for each compound was extracted into a new file. These were than sorted. ZINC compound files scoring better than $-7.3 \mathrm{kcal} / \mathrm{mol}$, with more negative scores being better, were included in the list in this manuscript as "hit" compounds.

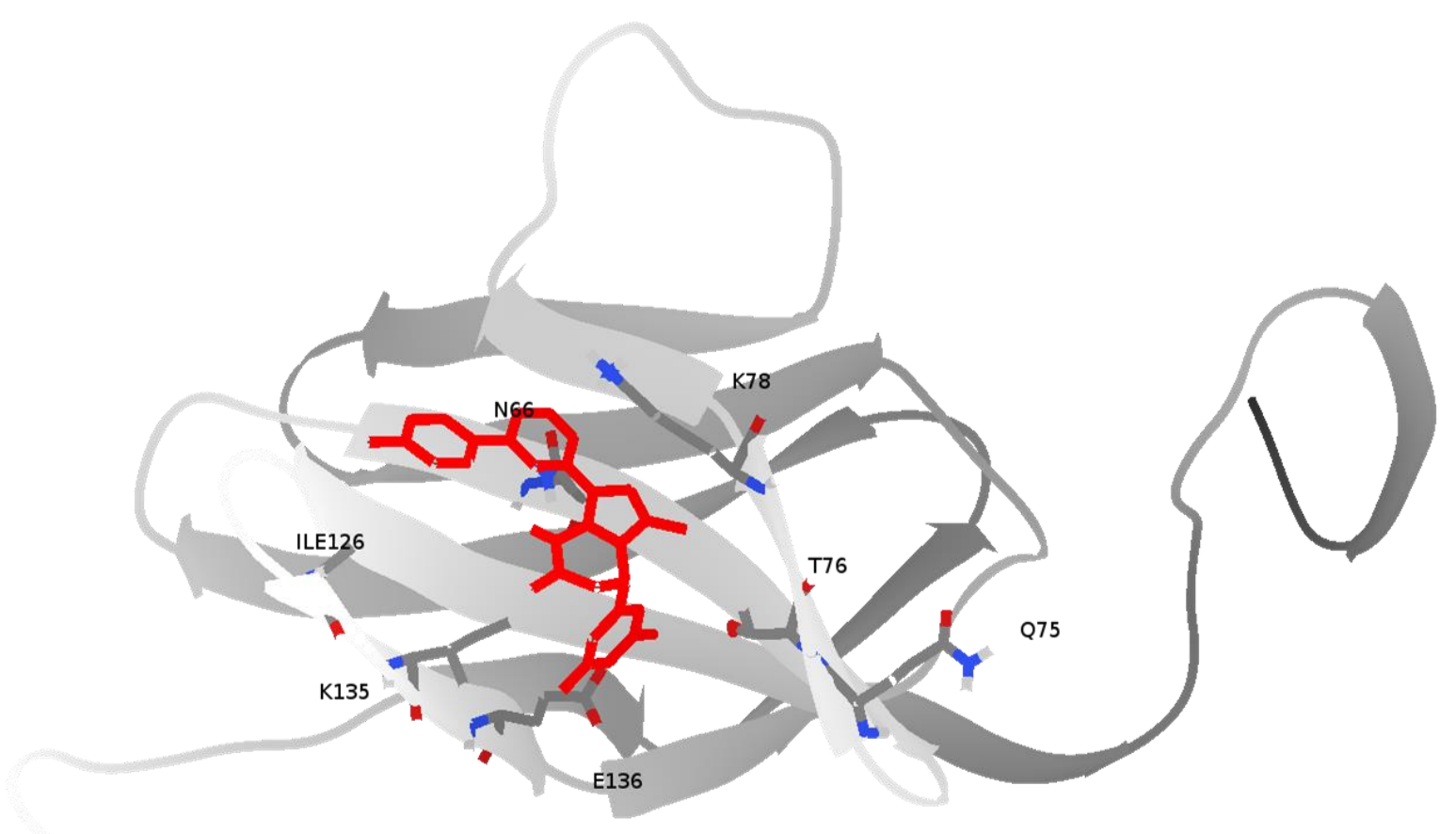

Figure 1. PD-1 with potential extracellularly binding inhibitor docked. ZINC data base entry ZINC15670145 (red) automatedly docked at the PD-L1 interface of apo PD-1 model. Selected residues in this interface have been shown in stick and have been labeled. 


\section{Results}

We operated on the presumption that docking scores are a rough, relative estimate of ligand binding affinity. Three "hit" compounds that scored better than $-7.3 \mathrm{kcal} / \mathrm{mol}$, ranging from -7.3 to $-7.6 \mathrm{kcal} / \mathrm{mol}$, when Clean Drug Like set 98 was screened in the POI were identified (Table 1). Thirty-six "hit" compounds were identified in Clean Drug Like set 94 when the set was screened in the POI, ranging in score from -7.3 to $-7.9 \mathrm{kcal} / \mathrm{mol}$ (Table1). The best scoring compound of the virtual screening was ZINC15670145 that scored $-7.9 \mathrm{kcal} / \mathrm{mol}$. It is pictured in its best docked pose in Figure 1, with selected PD-1 residues from the PD-1 / PD-L1 interface represented in stick. Information on obtaining ZINC compounds, such as those mentioned in this study, is available on the ZINC 12 Database pages (zinc12.docking.org). A screenshot of our best "hit" compound's ZINC page is shown in Figure 2.

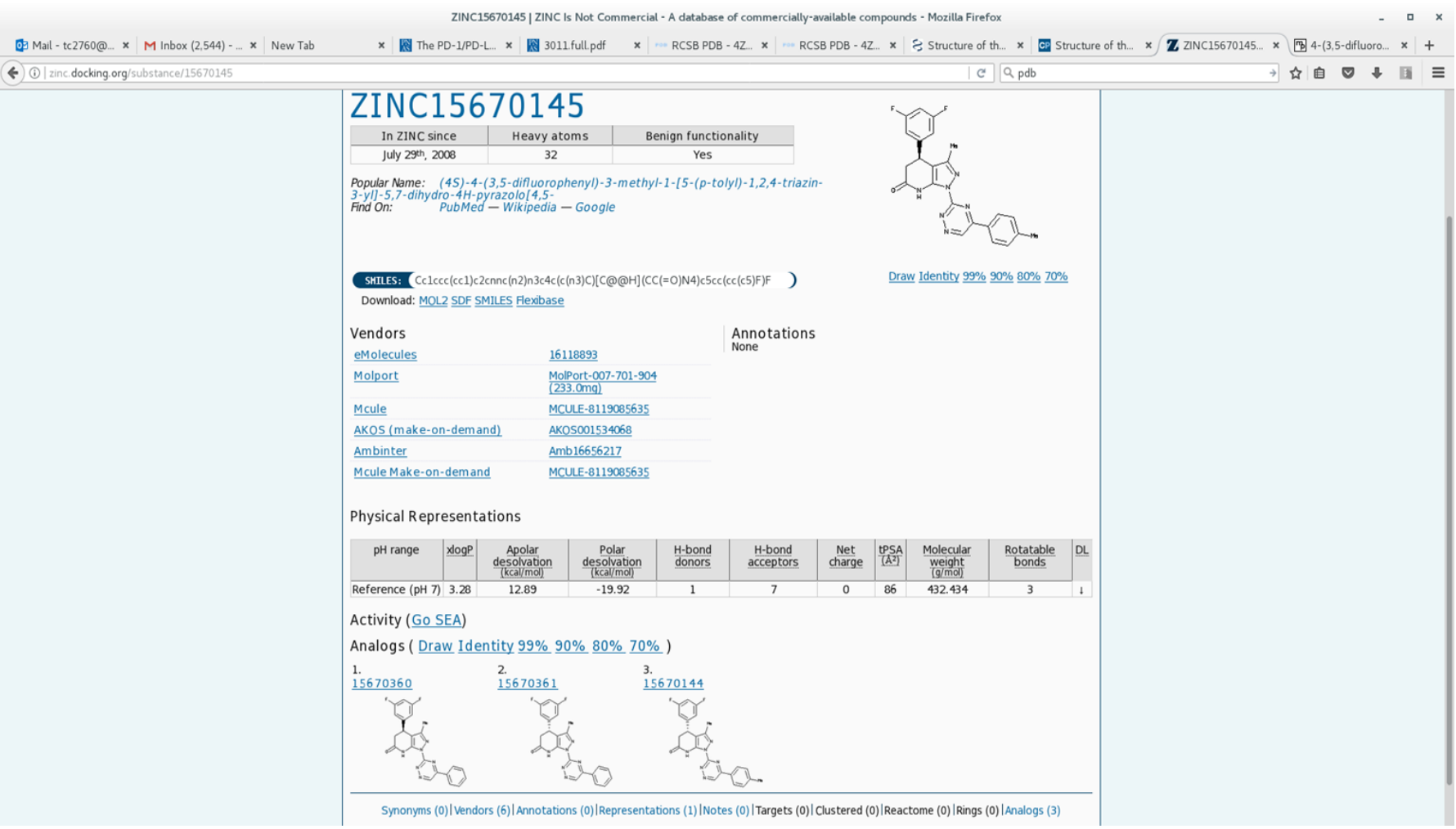

Figure 2. ZINC Database pages. Information and suppliers of the compound are available at the ZINC database (zinc12.docking.org)

\section{Table 1.}

Clean Drug Like 98 hits

score

ZINC24976988 $\quad-7.6$

ZINC15613537 -7.5

ZINC24976994 -7.3

Clean Drug Like 94 hits

$\begin{array}{lc} & \text { score } \\ \text { ZINC15670145 } & -7.9 \\ \text { ZINC25154768 } & -7.8 \\ \text { ZINC13396058 } & -7.7 \\ \text { ZINC19711040 } & -7.6 \\ \text { ZINC44448042 } & -7.6 \\ \text { ZINC15670077 } & -7.5 \\ \text { ZINC15670151 } & -7.5\end{array}$

Clean Drug Like 94 hits

(Continued 1) score

ZINC33809770 $\quad-7.5$

ZINC02633752 $\quad-7.4$

ZINC04985210 $\quad-7.4$

ZINC09849799 $\quad-7.4$

ZINC14464178 $\quad-7.4$

ZINC33140141 $\quad-7.4$

ZINC38932390 $\quad-7.4$

ZINC44448009 $\quad-7.4$

ZINC64859598 $\quad-7.4$

ZINC01376978 $\quad-7.3$

ZINC08607386 $\quad-7.3$

ZINC08619037 $\quad-7.3$

ZINC09723890 $\quad-7.3$

ZINC12099754 $\quad-7.3$

ZINC12133566 -7.3
Clean Drug Like 94 hits

(Continued 2) score

ZINC12886579 $\quad-7.3$

ZINC13075317 $\quad-7.3$

ZINC14967169 $\quad-7.3$

ZINC15670081 $\quad-7.3$

ZINC19374472 $\quad-7.3$

ZINC19761422 $\quad-7.3$

ZINC23873267 $\quad-7.3$

ZINC23997141 $\quad-7.3$

ZINC25089942 $\quad-7.3$

ZINC32906597 $\quad-7.3$

ZINC34786595 $\quad-7.3$

ZINC53517440 $\quad-7.3$

ZINC53517441 $\quad-7.3$

ZINC63160332 $\quad-7.3$ 


\section{Discussion}

The purpose of this communication is to make available a modest amount of data that would possibly otherwise go unused if not communicated now. This is done so that, maybe, someone can benefit from it. It is unlikely, at this time, that we will have an opportunity to experimentally test these theoretical computational findings so please feel free to cite this preprint if relevant. Thirty-nine compounds were identified that may possibly have the potential to inhibit PD-1 activation by PD-L1 via inhibiting the protein-protein interaction. ZINC15670145 appears to hold the greatest potential, of the "hit" compounds, to inhibit that interaction.

When testing compounds listed in this manuscript, it may be useful to begin testing with more than $10 \mu \mathrm{M}$ compound and to incubate the PD-1 expressing cells in the compound prior to adding PD-L1. A western blot for phosphorylated PD-1 or downstream phosphorylation may be an acceptable, semi quantitative test for compound inhibitory activity. Less phosphorylation when compound was added vs PD-L1 treated control would indicate inhibitory activity of that test compound.

Please note that the findings in this manuscript are entirely theoretical and there is no known experimental data existing on these findings. None of the listed compounds have been shown to be safe or effective immunotherapeutics. Under no circumstances should they be used as such without thorough and complete pharmaceutical development. The purpose of this manuscript is to offer a modest suggestion and potential starting point for such pharmaceutical development.

\section{References}

1. Sharma, P. and J.P. Allison, The future of immune checkpoint therapy. Science, 2015. 348(6230): p. 56-61.

2. Wu, X., et al., Application of PD-1 Blockade in Cancer Immunotherapy. Comput Struct Biotechnol J, 2019. 17: p. 661-674.

3. $\quad$ Keir, M.E., et al., PD-1 and its ligands in tolerance and immunity. Annu Rev Immunol, 2008. 26: p. 677-704.

4. $\quad$ Reck, M., et al., Pembrolizumab versus Chemotherapy for PD-L1-Positive Non-Small-Cell Lung Cancer. N Engl J Med, 2016. 375(19): p. 1823-1833.

5. Kimani, S.G., et al., Small molecule inhibitors block Gas6-inducible TAM activation and tumorigenicity. Sci Rep, 2017. 7: p. 43908.

6. Guzik, K., et al., Development of the Inhibitors that Target the PD-1/PD-L1 Interaction-A Brief Look at Progress on Small Molecules, Peptides and Macrocycles. Molecules, 2019. 24(11).

7. Trott, O. and A.J. Olson, AutoDock Vina: improving the speed and accuracy of docking with a new scoring function, efficient optimization, and multithreading. J Comput Chem, 2010. 31(2): p. 455-61.

8. Zimmermann, L., et al., A Completely Reimplemented MPI Bioinformatics Toolkit with a New HHpred Server at its Core. J Mol Biol, 2018. 430(15): p. 2237-2243.

9. Tan, S., et al., An unexpected N-terminal loop in PD-1 dominates binding by nivolumab. Nat Commun, 2017. 8: p. 14369.

10. Morris, G.M., et al., AutoDock4 and AutoDockTools4: Automated docking with selective receptor flexibility. J Comput Chem, 2009. 30(16): p. 2785-91.

11. Irwin, J.J. and B.K. Shoichet, ZINC--a free database of commercially available compounds for virtual screening. J Chem Inf Model, 2005. 45(1): p. 177-82.

12. Forli, S., et al., Computational protein-ligand docking and virtual drug screening with the AutoDock suite. Nat Protoc, 2016. 11(5): p. 905-19. 